\title{
Analysis of the Efficient Energy Prediction for 5G Wireless Communication Technologies
}

\author{
https://doi.org/10.3991/ijet.v14i08.10485
}

\author{
Haider Th. Salim AlRikabi $(\bowtie)$, Abdul Hadi M.Alaidi, \\ Ahmad Shaker Abdalrada, Faisal Theyab Abed \\ Wasit University, Wasit, Iraq \\ hdhiyabeuowasit.edu.iq
}

\begin{abstract}
With the growth of technological devices, gadgets and utility products in routine life, there is need to escalate the energy optimization with higher degree of accuracy and performance. Earlier the $4 \mathrm{G}$ networks were used are quite prominent and the wireless scientists are working ahead towards the direction of $5 \mathrm{G}$. In $5 \mathrm{G}$ based next-generation networks there are the projected features to transmit the huge amount of data and signals to the different locations whether to short or distant locations. The energy optimization, preservation and harvesting are key perspectives of research in advance gadgets in which the key focus is to minimize the energy loss and escalate the overall life period time of the network environment. These gadgets include assorted sensor nodes which communicate to each other using clustering and sharing of signals with the overall collaboration on the specific domain. In this manuscript, the mechanisms and methodologies for the energy parameter in the $5 \mathrm{G}$ networks are presented so that the greater accuracy and throughput can be obtained. In addition, a comparison has been established among the old classical network generations and the new 5G networks. The comparison is done predictably in terms of data rate, Latency, Mobility, Energy, and Efficiency of Spectrum. These specifications of the various network generations have been compared in order understand and highlight the benefits and advantages of the new coming generation $(5 \mathrm{G})$ over the features of the traditional network generations. Moreover, this paper is intended to show the challenges and related issues might be faced to achieve the implementation of the features and specifications of the new generation technology $(5 \mathrm{G})$. As a result, the new $(5 \mathrm{G})$ will be more efficient and effective in terms of high data transfer rate, low latency, Mobility, and Energy. This is very important because it draws a road map for many exciting technologies and infrastructures including Internet of Things (IOT), remote control of industrial machinery and robotics, and much faster download speed. Thus, 5G will support carrying huge amount of data faster, which will help to support smarter and reliable technology infrastructures and environment.
\end{abstract}

Keywords-5G, wireless communications, New Radio, Mechanisms Prediction of energy, River Formation Dynamics (RFD), Nature Inspired Approach (NIA), Chanel State Information (CSI). 


\section{Introduction}

The next-generation $5 \mathrm{G}$ based networks are the upcoming technologies in many countries as it is still under research because of assorted dimensions [1]. The newer 3GPP normal includes any network using the New Radio (NR) code. 5G New Radio will include lower frequencies, from six hundred $\mathrm{MHz}$ to six $\mathrm{GHz}$. However, the speeds in these lower frequencies are solely with modesty over new 4G systems, predicted at (15-50) percent faster [2-4]. The network environment based on the 5G technology is expected to have enormous features and resource optimized approach with the specifications of IMT-2020 [5].
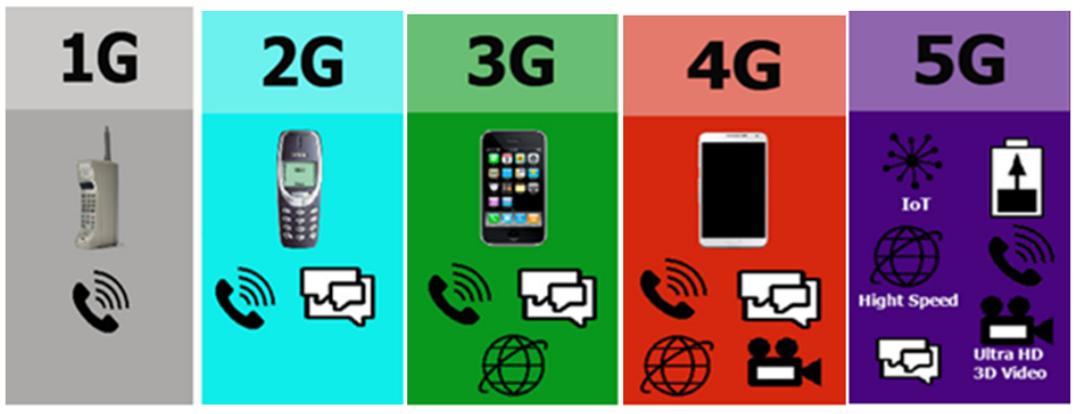

Fig. 1. Variants of Mobile Wireless Networks: $a-1 G ; b-2 G ; c-3 G ; d-4 G ; e-5 G$

Table 1. The prediction characteristic of $5 \mathrm{G}$

\begin{tabular}{|l|l|c|}
\hline \multicolumn{1}{|c|}{ Key Feature } & \multicolumn{1}{c|}{ Sketch } & Goal \\
\hline High transfer the data & Highest data rate that can be achieved & $20 \mathrm{Gbit} / \mathrm{s}$ \\
\hline Data rate at user end & Data rate that can be achieved at user end & $1 \mathrm{Gbit} / \mathrm{s}$ \\
\hline Latency & Radio network contribution to packet travel time & $1 \mathrm{~ms}$ \\
\hline Mobility & Maximum speed for handoff and QoS requirements & $500 \mathrm{~km} / \mathrm{h}$ \\
\hline Density of connection & Devices or Systems per unit area & $10^{6} / \mathrm{km}^{2}$ \\
\hline Energy & $\begin{array}{l}\text { Data Transmitted and Received per unit energy con- } \\
\text { sumption }\end{array}$ & Equivalent to 4G \\
\hline $\begin{array}{l}\text { Efficiency of Spec- } \\
\text { trum }\end{array}$ & Throughout per wireless bandwidth & $3-4 \mathrm{x} 4 \mathrm{G}$ \\
\hline Area traffic capacity & Traffic in the coverage area & $10(\mathrm{Mbit} / \mathrm{s}) / \mathrm{m}^{2}$ \\
\hline
\end{tabular}

5G networks are expected to be launched worldwide by year 2020 by different companies including Intel, Qualcomm, Lenovo, Nokia, Ericsson and many others.

\section{Analysis of Literature Data and Problem Statement}

One of the unresolved problems in $5 \mathrm{G}$ wireless communication is that how to provide huge information capacity with consideration of price, size and the efficiency for cellular. The architectures and technologies which leads to invent new modern communication technologies for $5 \mathrm{G}$ shows in [6]. Another problem mentions in [7] is how 
to proposed prediction method to predict Chanel State Information (CSI) based on proposed an online CSI scheme from historical data in $5 \mathrm{G}$. The main base station of $5 \mathrm{G}$ covers wide area with vary places. Although multi devices which has operated on the same frequency band, the CSI is different [7].

Predictability in dynamic RSS (Really Simple Syndication) and the patterns in spectrum bands studied by [8]. Security in $5 \mathrm{G}$ will be hard issue because the packet channel is allocated for basic packet channel however, it is used for huge range of applications. The connection is very important among these application and internet to integrate of user and industries. These application lead to revolution in technology used in various areas of life. For example, smart home, using IOT to manage and saving electrical energy, air pollution meter, smart trash collector, smart office system and etc. [9]. These advance technology applications require wide packet and super speed of communication. The main challenge will face us is that the demand of bandwidth will increase. On another hand, quality of service delivered to subscribers should be as good as expected [10-12]. In addition, the ray-tracing of losing routing prediction when use $5 \mathrm{G}$ trail and make simulation based on Vodafone's LTE [13]. The benefit of migrating to $5 \mathrm{G}$ mobile technology is shown in [14].

\section{The Purpose and Objectives of the Study}

The aim of the work is to minimize the energy losses and getting higher gain in the overall performance integrity so the overall network environment can be made more efficient.

To achieve this goal, the following tasks were set:

- Using Nature Inspired Approach (NIA)

- Proposed Algorithm to evaluate the existing protocol for energy optimization Then optimize the wireless network

- Measure the complexity of the proposed algorithm

- Evaluation the security of classical approach with NIA

- Using assorted cloud simulation scenario with varying number of nodes to log the result of packet data that travels in secure channel

\section{$4 \quad$ Energy Optimization using Nature Inspired Approach (NIA) in 5G Networks}

In the continuous years, the wireless networks with the energy usage issues [6, 7] are winding up more occupied with the investigation space. Besides the Internet of Things (IOT) [8] is furthermore winding up extraordinarily detectable in the similar area in which the valuable streamlining of advantages is required. In this examination work, the best approach to manage organize the nature moved approach is shown to achieve the more elevated amount of energy streamlining and protection with the general lifetime of the network correspondence. The key thought of the cluster head reworking using nature pushed blend of fragile handling and internal modules of en- 
ergy streamlining are imitated using recognizable test framework for impel wireless networks.

The key points of the work joins the perspectives that top level of change of energy using Server Selection and Shuffling for unprejudiced and execution careful approach. Dynamic topology with the objective that the consistency can be checked and surveyed.

Dynamic Hash Key based Transmission to keep up a vital separation from Energy Consuming Assaults and achieving power and energy careful transmission for raised lifetime. The use of metaheuristics or nature breathed life into procedures is reliably in research to achieve the larger amount of exactness. The work in this paper is focused on the use of River Formation Dynamics (RFD) based approach for 5G energy optimization [9].

The proposed approach is having more elevated amount of lifetime, accuracy and as a rule upgrade characteristics. Nature Inspired Approaches are extensively used for dealing with streamlining issues from a long time and that is the reason this estimation is grasped to be executed in the wireless networks.

The proposed approach is evaluated on various specifications including energy propelled, accuracy, turnaround time and when all is said in done execution of the network. The wireless center points with level of energy and lifetime are offered occasion to be cluster head so all around execution and period time of the clustered condition can be raised.

The diverse modules are completed in this work close by the time of dynamic biograph for center points and sensors in wireless condition. The period of insufficient grid for accessibility of the Wireless Nodes is the base points of view with the assignment of unpredictable energy level and edge regards for lifetime. The Implementation of cluster head decision in wireless sensor networks and examination of the executions of traditional and proposed approach on different center points is done in which the drive Energy Optimization in Wireless Networks reproduced condition. Reenactment and Implementation using Biograph apparatus compartment is locked in.

The Analytics of Server Shuffling for Energy Optimization and Implementation in the propagation gadget. The Advance Energy Optimization Avoidance of Energy Consuming Assaults is given the dynamic energy change and key exchange to veil the imposter wireless center points from the network to defend the energy and for the most part execution [10].

The investigation target in this work embodies the different concentrations including to survey the execution of classical approach for Energy Optimization in Assorted Scenarios. To complete the NIA-5G for Energy Optimization and Dynamic Server Selection in replicated condition and the Evaluation of the Results in Multiple Nodes and Scenarios. 


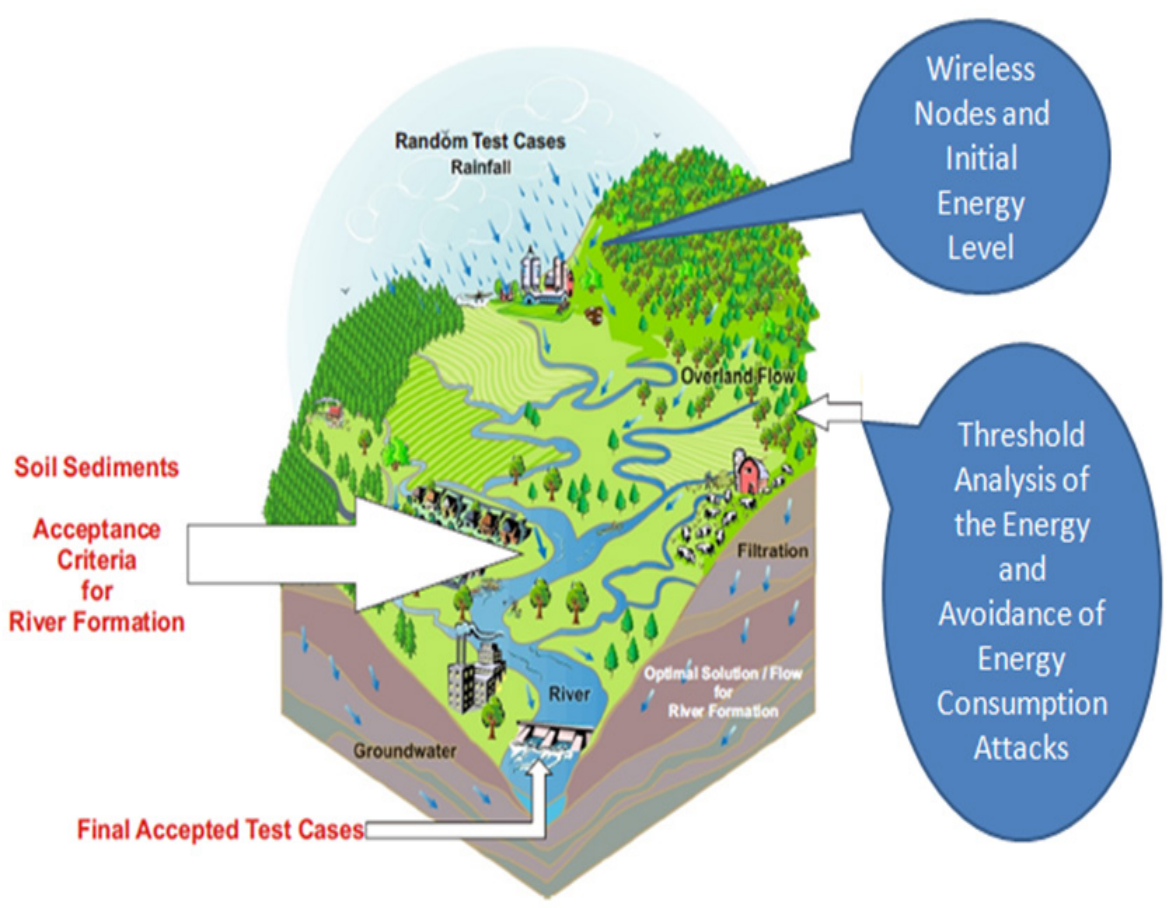

Fig. 2. Flow of NIA-5G

In the mathematical mode, the notations are specified as follows [11]:

$\mathrm{h}$ - Region of Finding the Best Fit Sediments (Parameters) from River (Global Search Space).

$\Delta$ - Diversion or Change in the Parameter Aspects using Fuzzy Mathematical Model. It is implemented to avoid the biasing and to improve the transparency.

$\phi-$ This variable parameter is directly proportional to $\Delta$. It means the value will change on the basis of changes on other parameter. This approach is used to maintain the consistency in the algorithmic approach. 


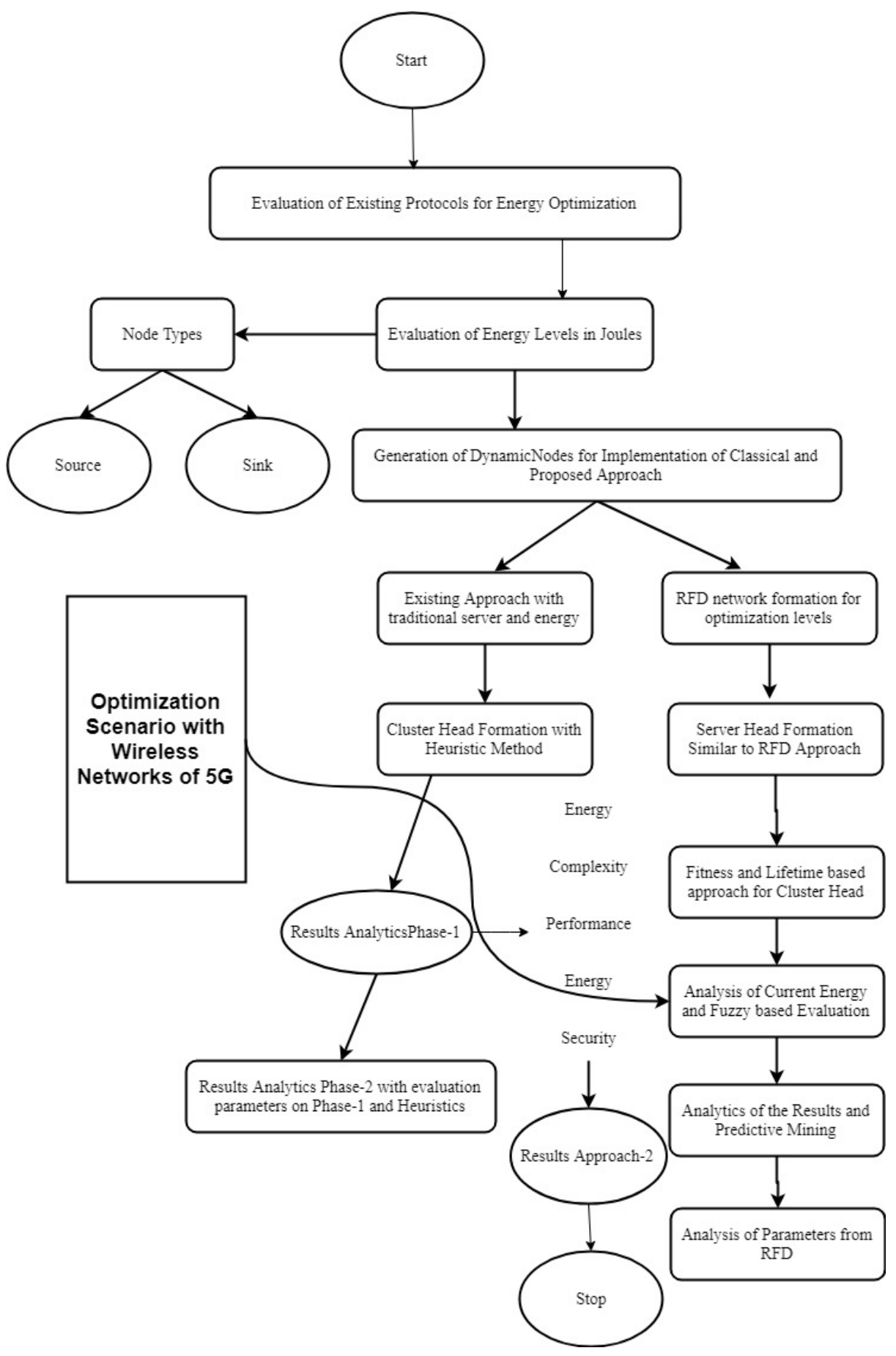

Fig. 3. Evaluation of Existing Protocols for Energy Optimization 


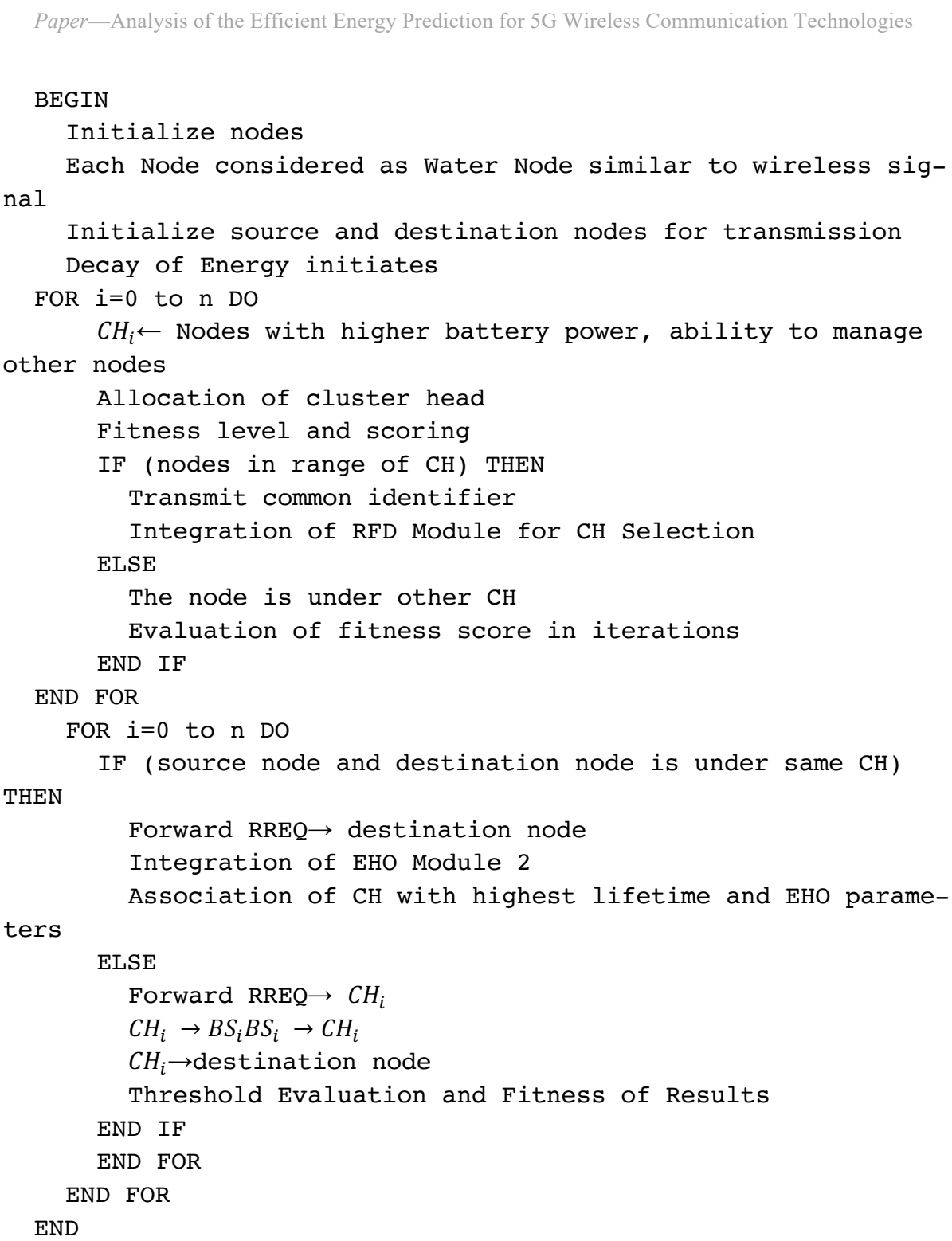

\section{$5 \quad$ Algorithm}

Initialization. Generation of nodes or wireless systems; Division of Water Drops as Population for swam based approach to n number of Groups or Clusters; Calculation of the Fitness Score with Each Individual; Setting up the Counter $\mathrm{i}=1$ and Maximum Value of Population in Current Cluster MaxPopulation 


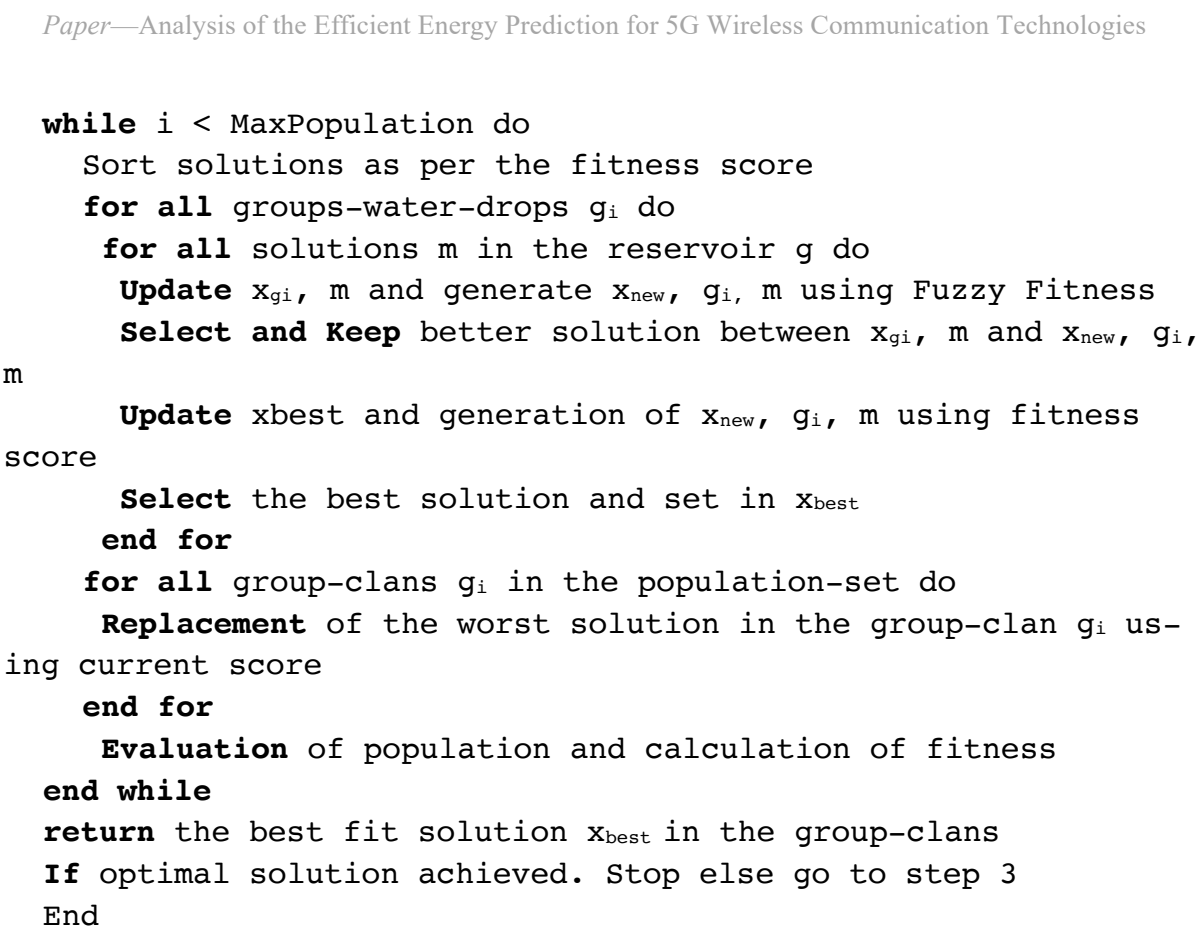

\section{Importance of Novel Approach}

- Higher degree of optimization with the threshold analysis of multiple systems so that the server selection can be dynamic.

- The shuffling or changing of the cluster head to avoid any type of attack to disguise the cracker of intrusion attempts.

- The nature inspired approaches are always effectual in terms of minimum delay and higher performance in the multiple scenarios.

- The repeated flow in the path makes sure the genuine or authentic river formation or simply network path for traversing of the data packet in secured channel

- The following results are logged using assorted cloud simulation scenarios with the varying number of wireless sensor nodes. The simulations based on different sets of input parameters are executed so that the overall energy, integrity and consistency of classical and novel projected approaches can be evaluated in effectual methodology (Table 2). 
Table 2. Evaluation of Energy Parameter with each approach

\begin{tabular}{|c|c|c|}
\hline Simulation Scenario with Varying Input Sets & Traditional & NIA-5G \\
\hline 1 & 1312 & 2712 \\
\hline 2 & 1232 & 2361 \\
\hline 3 & 1114 & 2294 \\
\hline 4 & 1190 & 2970 \\
\hline 5 & 1277 & 2353 \\
\hline 6 & 1795 & 2604 \\
\hline 7 & 1473 & 2093 \\
\hline 8 & 1277 & 2692 \\
\hline 9 & 1850 & 2043 \\
\hline 10 & 1080 & 2934 \\
\hline 11 & 1413 & 2585 \\
\hline 12 & 1371 & 2153 \\
\hline 13 & 1000 & 2482 \\
\hline 14 & 1035 & 2791 \\
\hline 15 & 1331 & 2754 \\
\hline 16 & 1821 & 2135 \\
\hline 17 & 1683 & \\
\hline & & \\
\hline
\end{tabular}

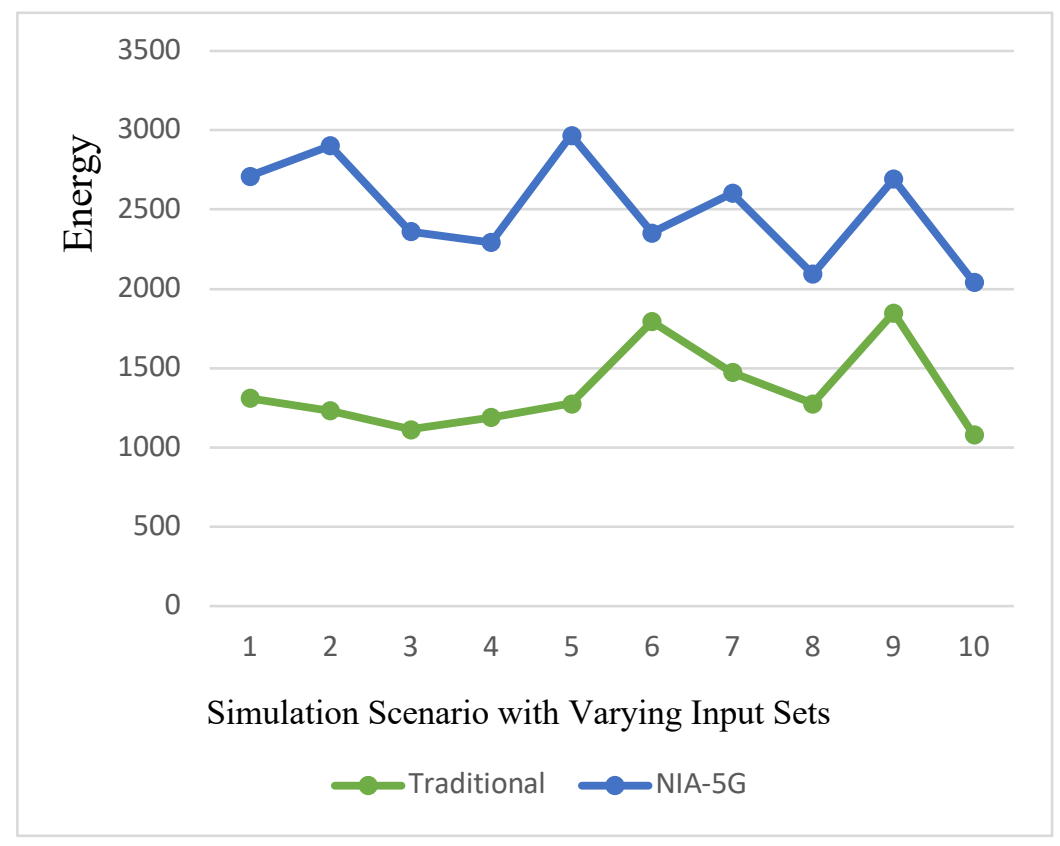

Fig. 4. Evaluation of Energy Parameter with each approach

As security and integrity are the key focus in this research manuscript, the following results are evaluated from the analytics of security in the projected approach. Security is analyzed from each simulation scenario of cloud implementation so that 
overall vulnerabilities can be avoided with the escalation of security and cumulative performance of projected approach.

The research work is having the integration of security as the overall energy optimization is associated with the security perspectives. Many times, the gadgets consume and deplete the energy to huge level and that is required to be associated (Table 3, Fig. 4).

Table 3. Evaluation of Security Aspects

\begin{tabular}{|c|c|c|}
\hline Simulation Attempt & Classical Approach & NIA-5G \\
\hline 1 & 10 & 37 \\
\hline 2 & 11 & 33 \\
\hline 3 & 15 & 34 \\
\hline 4 & 15 & 38 \\
\hline 5 & 14 & 40 \\
\hline 6 & 15 & 39 \\
\hline 7 & 17 & 33 \\
\hline 8 & 18 & 33 \\
\hline 9 & 12 & 41 \\
\hline 10 & 10 & \\
\hline
\end{tabular}

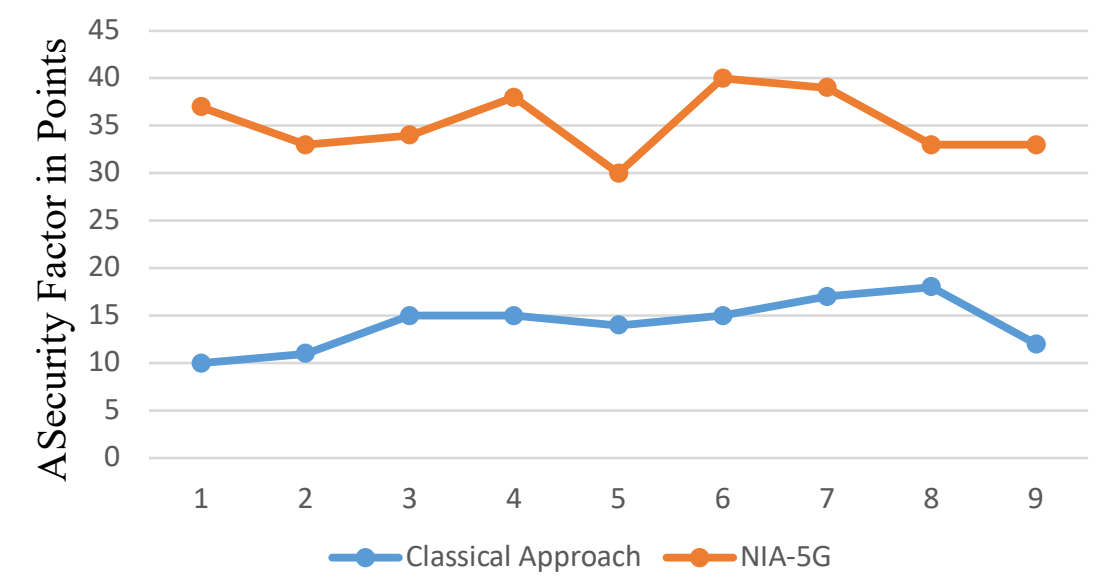

Simulation Attempt or Scenario with Varying Nodes

Fig. 5. Evaluation of Security Aspect. 


\title{
7 Formulation of Parameters (Previous Approach)
}

\subsection{Time Complexity: Big-O N (n log (n2))}

Creation of Solutions and Traversal Paths with Performance Optimization Based Performance Big-O(n) + Evaluation of the Requirements and Resource Replenishment Big-O (n $\log n)+$ Allocation of data to the Controllers Big-O (n) + Controllers with loop towards allocation of resources to Base Controller Big-O (n2) + Base Controller and Dataset Interfacing Big-O (n) + Fetching of Required Parameters for best fit allocation Big-O (n) + Sequential Assignment and Processing Big-O (n) + Analysis of factors Big-O (n2) + Inner Loop for Assignment of records and key features Big-O (n2) + Deep Inner Loop for record replenishment $n$ Log (n) + Time and frequency based job execution $n \log (n 2)+$ Job preparation and Execution on server $n \log (n)=$ $\mathrm{Big}-\mathrm{O}(\mathrm{n} 2)+\mathrm{n} \log (\mathrm{n})$.

\subsection{Complexity}

Tasks and Resources Requirements (n2) + Memory Allocation for Tasks Big-O (n2) + Memory Allocation for Paths $\mathrm{n} *$ Big0N (n) + Inner Requirements for Solutions $\mathrm{n} * \operatorname{Big} 0 \mathrm{~N}(\mathrm{n})+$ Performance Consumption at each phase Big0N(n) + Deep Inner Performance Consumption $\mathrm{n} \log (\mathrm{n})+$ Cumulative Performance Allocation $\mathrm{n}$ * $\operatorname{Big} 0 \mathrm{~N}(\mathrm{n})=\operatorname{Big}-\mathrm{O}\left(\mathrm{n}^{2}\right)$.

\subsection{Performance}

\author{
$\mathrm{r}: \mathrm{n} *(1 / \mathrm{t}) * \mathrm{RNDFCT}$ \\ $\mathrm{n}$ : Length of the Input \\ $\mathrm{t}$ : Execution Time \\ RNDFCT: Random Fuzzy Random
}

\section{Proposed Approach}

\subsection{Time complexity: Big-O (n2)}

Creation of Solutions and Optimized Based Performance + Evaluation of the Requirements and Resource Replenishment + Controllers with loop towards allocation of resources to Base Controller + Base Controller and Dataset Interfacing + Allocation of Tasks to the Controllers and machine learning module+ Fetching of Required Parameters for best fit allocation + Sequential Assignment and Processing + Analysis of factors+ Inner Loop for data Assignment and Performance+ Deep Inner Loop for replenishment + Time and frequency based job execution + Job preparation and Execution on server : $\mathrm{Big} 0 \mathrm{~N}(1)+\operatorname{Big}-\mathrm{O}(\mathrm{n})+\operatorname{Big}-\mathrm{O}(\mathrm{n} 2)+\mathrm{Big}-\mathrm{O}(\mathrm{n})+\mathrm{Big}-\mathrm{O}(\mathrm{n})+\mathrm{Big}-$ 
$\mathrm{O}(\mathrm{n})+\operatorname{Big}-\mathrm{O}(\mathrm{n} 2)+\operatorname{Big}-\mathrm{O}(\mathrm{n} 2)+\mathrm{n} \log (\mathrm{n})+\mathrm{n} \log (\mathrm{n})+\mathrm{n} \log (\mathrm{n}): \operatorname{Big}-\mathrm{O}(\mathrm{n} 2)+\mathrm{n}$ $\log (n)$

\subsection{Complexity}

Tasks and Machine Requirements + Memory Allocation for Virtual Tasks + Memory Allocation for Data Features+ Inner Requirements for key features+ Memory Consumption at each phase + Deep Inner Memory Consumption + Cumulative Space Allocation $=$ Big-O (n) + Big-O (n) $+n *$ Big-O (n) $+n *$ Big-O (n) + Big-O (n) $+n$ $\log (\mathrm{n})+\mathrm{n} * \operatorname{Big}-\mathrm{O}(\mathrm{n})=\operatorname{Big}-\mathrm{O}(\mathrm{n})$

\subsection{Performance}

\section{$\mathrm{r}: \mathrm{n} *(1 / \mathrm{t}) * \mathrm{RNDFCT}$ \\ $\mathrm{n}$ : Length of the Input \\ $\mathrm{t}$ : Execution Time}

RNDFCT: Random Fuzzy Random

Portability (p) and Efficiency (e) is directly proportional to reliability

Cost factor $(\mathrm{c}): \mathrm{c}: 1 /(\mathrm{r} *(\mathrm{p}+\mathrm{e})) * 100$

Reusability $(\mathrm{ru})=\mathrm{r}$

\section{$9 \quad$ Energy and the Environment Factors}

Energy and Environment investigate bases on the age, storing and capable use of imperativeness and typical resources and the evaluation of the coordinated effort amongst nature and essentialness progresses with a particular ultimate objective to develop immaculate and manageable power source. Research on the cooperation between practical power source progressions and the earth (standard resources) is crucial to appreciation and working up sensibility. This investigation epitomizes the interrelation among air and regular conditions and the creation and utilization of impeccable, reasonable power source. Research on upgraded sun arranged, wind, and bioessentialness, and the coordinated effort of these headways with water resources, atmosphere, and air, describes this center intrigue. Two subjects are connected with this key research area: Efficient and Clean Energy Systems and Sustainable Environmental Systems. Research related to this theme can develop the current technologies to increase the potency of assorted emphasized energy systems, whereas minimizing the potential environmentally disruptive aspect effects of those technologies.

In addition, Research is needed to better understand each ecosystem's components, functions, and processes for sustainability, evolution, restoration, and conservation, as it interfaces with renewable energy production and its socio-economic impacts. 


\section{Discussion of the Research Results}

The advantages of our research is to highlight the importance and efficiency of the $5 \mathrm{G}$ networks. It shows how RFD can be utilized for the $5 \mathrm{G}$ energy optimization. The results obtained from the simulation scenarios for the Novel approach compared to the traditional approach shows a high level of security and integrity obtained of applying the Novel proposed approach. In addition, high performance as well as low delay in data transfer has been noticed comparing to the classical methodologies. The simulation performed in this research showed high level optimization as the server is being picked by multiple systems dynamically. In addition, the security and integrity features were perfect as the changing of the cluster head is applied in order to prevent any attack type. Therefore, the proposed approach can ensure fast and reliable 5G networks schema. The advantages of these modern networks include high-speed data transfer reach to Gigabits with low latency, and optimized energy levels. In addition, it allows for clean energy consuming and cleaner environment for communications and digital operations. The new approach schema for $5 \mathrm{G}$ networks can be beneficial in many areas and fields. Many applications require high-speed data transfer, reliable and available connectivity, secure and integrity communication channels. These applications include visualization of universe and galaxies, remote treatment of patients (remote surgery applications), fast and accurate detection of natural disasters, and easy locating of a missing person.

\section{Conclusion}

- The Nature Inspired Approach (NIA) has helped to minimize the level of energy losses by utilizing the proposed methodology and algorithm.

- The proposed algorithm is applied to measure the efficiency level of the energy optimization protocol.

- The proposed approach achieved higher gain in terms of network performance, integrity, higher data transfer rate, and higher level of security.

- This research helped to compare and measure the security levels of the classical approach as well as the NIA approach. The proposed method indicates high level of secure communications due to the low energy loss and high gain in performance and integrity.

- The results obtained showed less data transfer delay, high data transfer rate, accurate data transmission, and high levels of security and integrity. These results all have been obtained through the implementation of cluster head decision and the server shuffling techniques, which helped to achieve the energy levels optimization.

- Finally, the proposed approach helps to build a fast, secure, and reliable network infrastructure that supports many time-critical applications and systems such as remote industry robotics, remote patient treatment, and space monitoring and discovering systems. Therefore, the new approach helps to implement 5G network 
schema that maintains security, availability, high speed, low latency, and optimized energy levels for its users (applications, systems, other infrastructures).

\section{References}

[1] Li, Q.C., Niu, H., Papathanassiou, A.T. and Wu, G., "5G network capacity: Key elements and technologies", IEEE Vehicular Technology Magazine, 2014, 9(1), pp.71-78. https://doi.org/10.1109/MVT.2013.2295070

[2] Al-Dulaimi, A. and Hu, R.Q., "New Considerations for 5G Wireless Systems" (From the Guest Editors), IEEE Vehicular Technology Magazine,2018,13(2), pp.4-6. https://doi.org/10.1109/MVT.2018.2813532

[3] Chopra, G., Jha, R.K. and Jain, S., 2018, January. "Security issues in ultra-dense network for 5G scenario" In "Communication Systems \& Networks (COMSNETS), 2018 10th International Conference IEEE", (pp. 510-512).

[4] Osseiran, A., Boccardi, F., Braun, V., Kusume, K., Marsch, P., Maternia, M., Queseth, O., Schellmann, M., Schotten, H., Taoka, H. and Tullberg, H., 2014, "Scenarios for 5G mobile and wireless communications: the vision of the METIS project, IEEE Communications Magazine, 52(5), pp.26-35. https://doi.org/10.1109/MCOM.2014.6815890

[5] Wang, T., Li, G., Ding, J., Miao, Q., Li, J. and Wang, Y., "5G spectrum: Is China ready?" IEEE Communications Magazine, 2015, 53(7), pp.58-65. https://doi.org/10. 1109/MCOM.2015.7158266

[6] Zappone, A., Debbah, M. and Altman, Z., Online energy-efficient power control in wireless networks by deep neural networks. In "IEEE Workshop on Signal Processing Advances in Wireless Communications (SPAWC) ", 2018. https://doi.org/10. 1109/SPAWC.2018.8445857

[7] Raghunathan, V., Schurgers, C., Park, S. and Srivastava, M.B., Energy-aware wireless microsensor networks. "IEEE Signal processing magazine", 2002, 19(2), pp.40-50. https://doi.org/10.1109/79.985679

[8] Rabanal, P., Rodríguez, I. and Rubio, F., September. Finding minimum spanning/distances trees by using river formation dynamics. In "International Conference on Ant Colony Optimization and Swarm Intelligence ",2008, (pp. 60-71). Springer, Berlin, Heidelberg. https://doi.org/10.1007/978-3-540-87527-7 6

[9] Gubbi, J., Buyya, R., Marusic, S. and Palaniswami, M. "Internet of Things (IoT): A vision, architectural elements, and future directions. Future generation computer systems, 2013,29(7), pp.1645-1660. https://doi.org/10.1016/j.future.2013.01.010

[10] Hu, R.Q. and Qian, Y., An energy efficient and spectrum efficient wireless heterogeneous network framework for 5G systems. "IEEE Communications Magazine", 2014, 52(5), pp.94-101. https://doi.org/10.1109/MCOM.2014.6815898

[11] Chopra, G., Jha, R. K., \& Jain, S. (2018, January). Security issues in ultra-dense network for 5G scenario. In 2018 10th International Conference on Communication Systems \& Networks (COMSNETS) (pp. 510-512). IEEE.

[12] Bukvić, M., Stanojević, B., \& Stanojević, M. (2018, May). Multiple objective channel allocation problem in 5G networks. In 2018 7th International Conference on Computers Communications and Control (ICCCC) (pp. 162-168). IEEE.

[13] Smiljkovikj, K., Elshaer, H., Popovski, P., Boccardi, F., Dohler, M., Gavrilovska, L., \& Irmer, R. (2014). Capacity analysis of decoupled downlink and uplink access in 5G heterogeneous systems. arXiv preprint arXiv:1410.7270. 
[14] Gupta, S., Bhardwaj, S. and Bhatia, P.K., A reminiscent study of nature inspired computation, "International Journal of Advances in Engineering \& Technology", 2011, 1(2), p.117.

\section{Authors}

Haider Th. Salim AIRikabi is a lecturer in the Engineering College/ Electrical Engineering Department at the Wasit University, Iraq, M.SC from California State University / Fullerton/USA. His area of research focuses on wireless communications, control systems and their applications.

Abdul Hadi M. Alaidi is a lecturer in the Engineering College, at the Wasit University, Iraq. His area of research focuses on algorithm and image processing.

Ahmed Shaker Abdalrada is a lecturer in the Engineering College, at the Wasit University, Iraq. His area of research focuses on information systems and Bioinformatics.

Faisal Theyab Abed is a lecturer in the Engineering College, at the Wasit University, Iraq. His area of research focuses on power systems and their applications.

Article submitted 2019-01-15. Resubmitted 2019-03-20. Final acceptance 2019-03-20. Final version published as submitted by the authors. 